\title{
MUD DEPOSITION SIMULATION AT THE CRFM OF AN AUTOMOBILE USING CFD SIMULATION
}

Filipe Fabian Buscariolo ${ }^{1}$, Julio Cesar Lelis Alves², Leonardo José Della Volpe ${ }^{2}$

${ }^{1}$ GM Brazil / NDF-USP

${ }^{2}$ GM Brazil

E-mail: filipe.buscariolo@gm.com, julio.alves@gm.com, leonardo.volpe@gm.com

\begin{abstract}
CFD is becoming very popular among the industries and the use of multiphase simulations is also increasing with more powerful CPUs and reliable CFD codes. The scope of this work is to present a mud deposition simulation methodology using CFD multiphase analysis at the CRFM of an automobile, in order to prevent low performance on the condenser or on the radiator and compromise the heat exchange performance. Mud reaches the front end of the car and results show the mud path and mud deposition on the CRFM and the blocked area.
\end{abstract}

Keywords: CFD (computational simulation), Mud simulation (multiphase simulation), CRFM.

\section{INTRODUCTION}

CFD simulations became a good solution to predict performance in a fluid system considering the growth of computational power. This fact also made possible to perform more complex simulations in a production response time, such as multiphase fluid simulations, involving two or more fluids.

Basically, multiphase CFD simulations can be divided into two groups, according to BUSCARIOLO and VOLPE (2014) [2]:

- Reactive: fluids interact chemically or physically to generate new products and properties changes. Example: combustion.

- Non-Reactive: two or more fluids are present in the computational domain, however they do not interact chemically or physically. Example: water ingestion simulation.

The present paper provides an example to the growing trend of using multiphase simulations in the industry. The main purpose is, by applying computational simulation, to identify mud path and deposition when the vehicle is crossing a 300mm mud height before any prototype is built, aiming to improve the vehicle's development cost and time and improve CRFM performance on off-road conditions. 


\section{OBJECTIVES}

Based on the real off-road condition of a car crossing certain mud height, in this case $300 \mathrm{~mm}$, at $20 \mathrm{kph}$, the present work was developed aiming to reproduce this condition virtually. An example of a vehicle in off-road conditions with mud is shown on Figure 1 below.

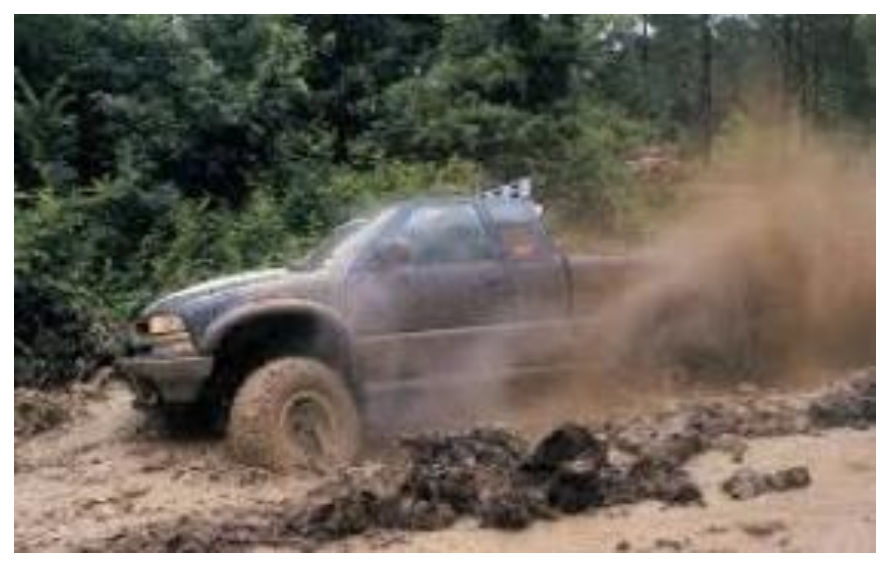

Figure 1. Vehicle in off-road conditions with mud.

The main objective of this paper is to identify mud path over the CRFM - Condenser, Radiator, Fan Module, analyze if there is any kind of deposition due to the mud by using a multiphase CFD simulation.

\section{METHODOLOGY}

The methodology proposed to this work is based on BUSCARIOLO and VOLPE (2014) [2] work, using correlation of the physical test with proposed multiphase CFD simulation for water path but now replacing the fluid by mud. In this case, the mud deposition will be analyzed only virtually.

In order to reproduce the car trying to cross a mudded area based on a test considering the car moving at $20 \mathrm{kph}$ cross a water height of $300 \mathrm{~mm}$, mud simulation was performed considering a production car at the same conditions with vehicle speed of $20 \mathrm{kph}$ and now mud height of $300 \mathrm{~mm}$ were applied to the multiphase CFD simulation, however in this case, the vehicle remains static and the mud and the air moves toward it. A schematic representation of the tunnel inlet is shown below. 


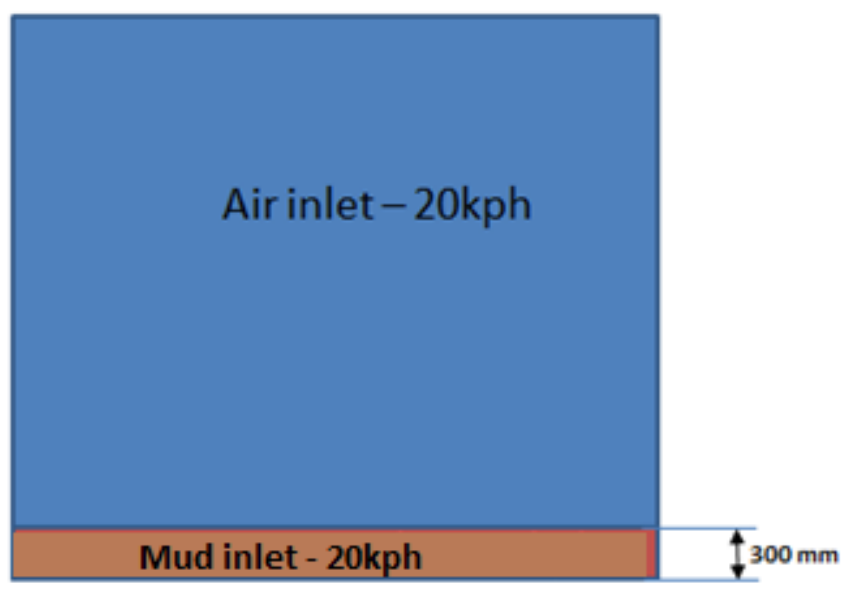

Figure 2. Wind tunnel inlet

CFD simulations were performed considering half of regular production car, in order to save computational time due to the main focus on the AIS, similar to approach used by BUSCARIOLO and VOLPE (2014) [2]. The simulation considered vehicle with rotating wheels, CRFM drop pressures and full underhood representation, assembled in a virtual Wind Tunnel, with dimensions similar to GM Wind Tunnel [6], once it has width similar to regular a street. Parameters considered in this work are show on table 1.

Table 1. Parameters used for CFD simulations

\begin{tabular}{|l|c|}
\hline Wind Tunnel Structure & General Motors (similar) \\
\hline Air Speed & $20 \mathrm{~km} / \mathrm{h}$ \\
\hline Outlet Pressure & Atmospheric Pressure \\
\hline Turbulence Intensity & $0.60 \%$ \\
\hline Boundary Layer Suction & Beginning of the Test \\
\hline Test Section Dimensions & $(5.4 \times 10.4 \times 10.0) \mathrm{m}$ \\
\hline Turbulence Model & $\mathrm{k}-\varepsilon$ \\
\hline
\end{tabular}

The ground simulation for this paper is moving ground with rotating wheels as mentioned before, which is the condition close to the real operating condition, according to [1], [2], [7] and [9] and the turbulence model considers swirl flow due to mud perturbations.

\section{Model Additional Considerations}

Based on BUSCARIOLO and VOLPE (2014) [2] work, for the model configuration of the multiphase, it was considered the Volume of Fluid (VOF) method which is a numerical technique for tracking and locating the free surface. It belongs to the class of Eulerian methods which are characterized by a mesh that is either stationary or is moving in a certain prescribed manner to accommodate the evolving shape of the interface.

VOF is an advection scheme-a numerical recipe that allows the programmer to track the shape and position of the interface, but it is not a standalone flow solving algorithm. The Navier-Stokes equations describing the motion of the flow have to be solved separately. [3] 
The turbulence model considered was the K-epsilon (k- $\varepsilon$ ) which is the most common model used in Computational Fluid Dynamics (CFD) to simulate turbulent conditions. It is a two equation model which gives a general description of turbulence by means of two transport equations. The first transported variable determines the energy in the turbulence and is called turbulent kinetic energy $(\mathrm{k})$. The second transported variable is the turbulent dissipation (epsilon) which determines the rate of dissipation of the turbulent kinetic energy.

The simulation was set as a transient with a fixed time step lower enough to the simulation reach convergence and represent the flow path. The total simulated time is calculated by dividing the domain length, which is $10 \mathrm{~m}$ in this case, by the speed of $20 \mathrm{kph}$ which gives a result of around 2 seconds to be simulated. In order to have stable flow, total simulated time was 4 seconds, same time used for water simulation on previous work.

\section{RESULTS}

The proposed methodology and model configuration based on water flow simulation were capable to reproduce a multiphase flow of a vehicle crossing a $300 \mathrm{~mm}$ mud height at constant speed of $20 \mathrm{kph}$ in a production level response time.

Results show good correlation with the water path over the car body as shown below:

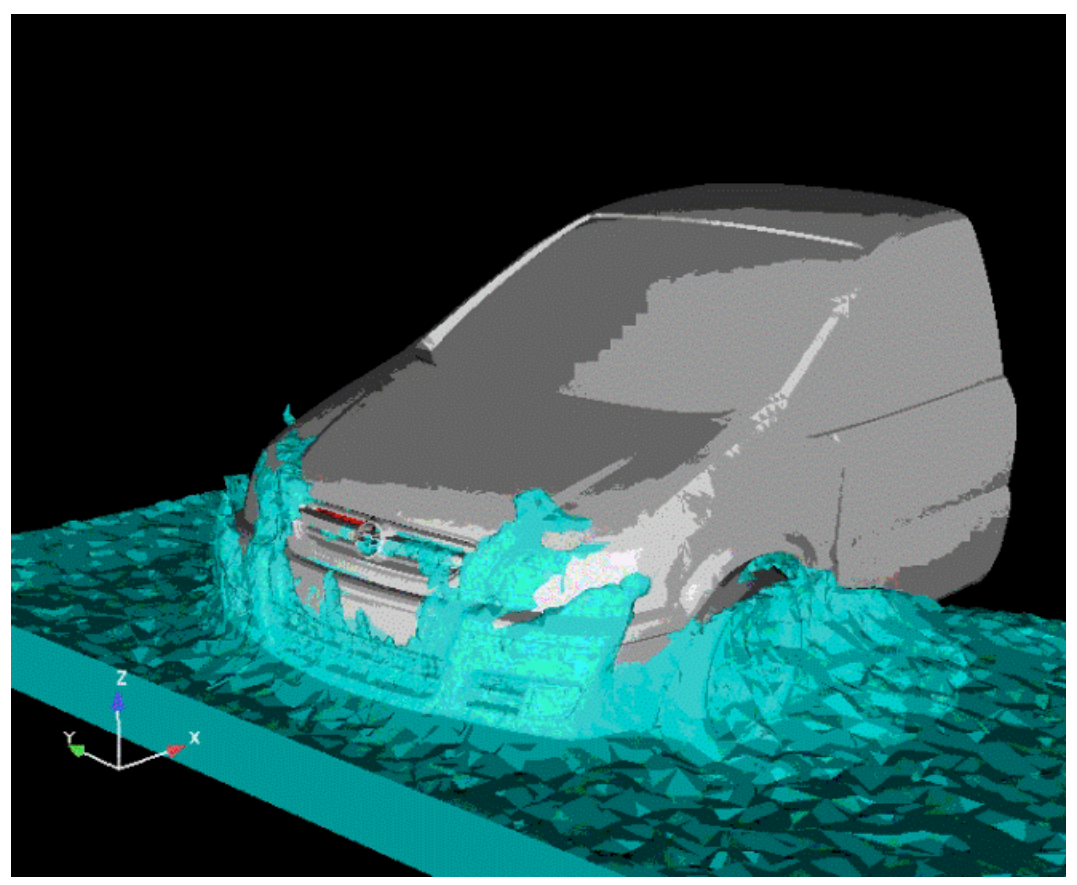

Figure 3. Water path on a production car-multiphase CFD Simulation 


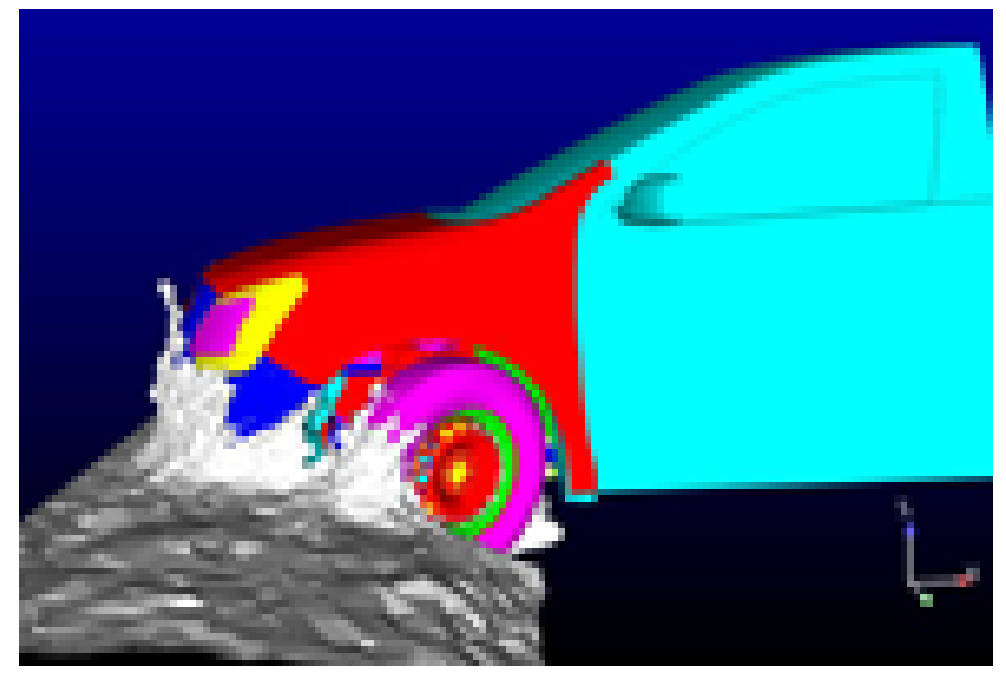

Figure 4. Mud path on a production car-multiphase CFD Simulation

\section{CONCLUSION}

The Industry is always seeking for solutions to improve vehicles development cost and time. The use of computational simulations is becoming a common practice inside the industries nowadays. Considering the hardware and software improvements among the years, the level of complexity of the Computer Aided Engineering (CAE) simulations are also growing.

As mentioned before, the use of CFD simulations are also increasing among automakers in order to improve vehicle's development time and also save cost with physical tests.

With more powerful CFD codes and, together with the increment of the number of processors, multiphase analysis, which are fluid simulations that consider two or more fluids in the same domain, become a nice solution and in a reliable production time.

In the present work, a methodology to perform a multiphase CFD simulation of a car crossing a mud height of $300 \mathrm{~mm}$ at $20 \mathrm{kph}$, which represents an off-road condition, was presented with good correlation results to the water physical test from reference [2].

Once the main focus of the paper was the CRFM, only half of the car was considered in order to speed up results and solution. Model was set considering VOF approach and K-epsilon turbulence model. Total simulated time was 4 seconds in a transient condition.

The methodology was capable to reproduce the mud splash effect on the vehicle crossing it, as shown before. This fact is really important once this approach can be used at early stages of the project to evaluate part performance and also save money by improving the design and performance of certain parts before any physical test. 


\section{CRFM Mud Deposition Analysis and Conclusions}

By analyzing the CRFM performance, which was the focus of this paper, computational simulation predicted mud deposition on the front of the condenser as shown on Figure 5, which was taken from the virtual test at 4 seconds of the total simulation time.

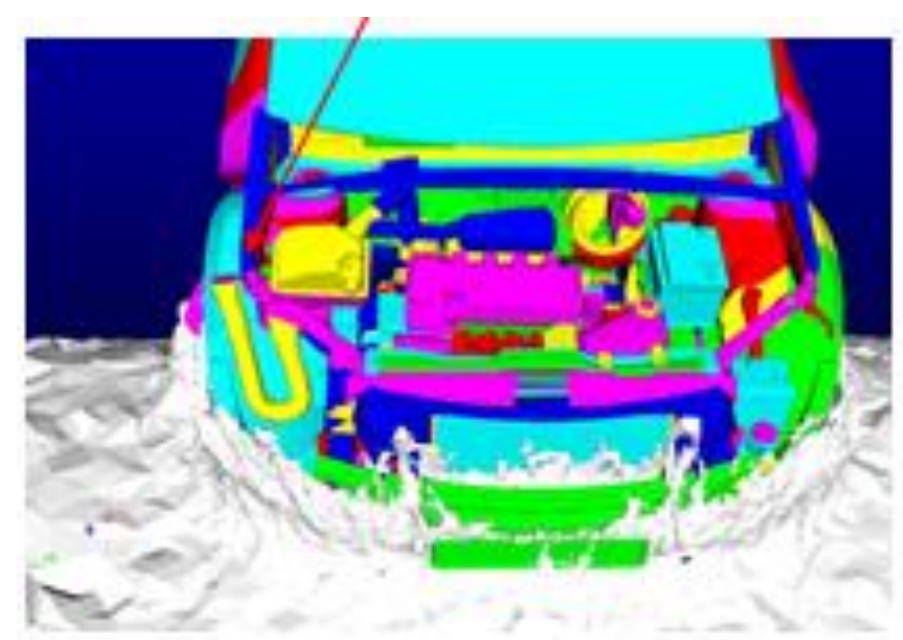

Figure 5. Mud deposition at the condensert on the simulation at 4 seconds of the simulation

Mud came from several gaps on the front end of the vehicle to the underhood and CRFM. An important fact is that the virtual model does not consider duck bills, which prevent mud penetration from the underbody. The side mud penetration due to the lack of duckbill is shown on Figure 6 at simulation time of 4 seconds.

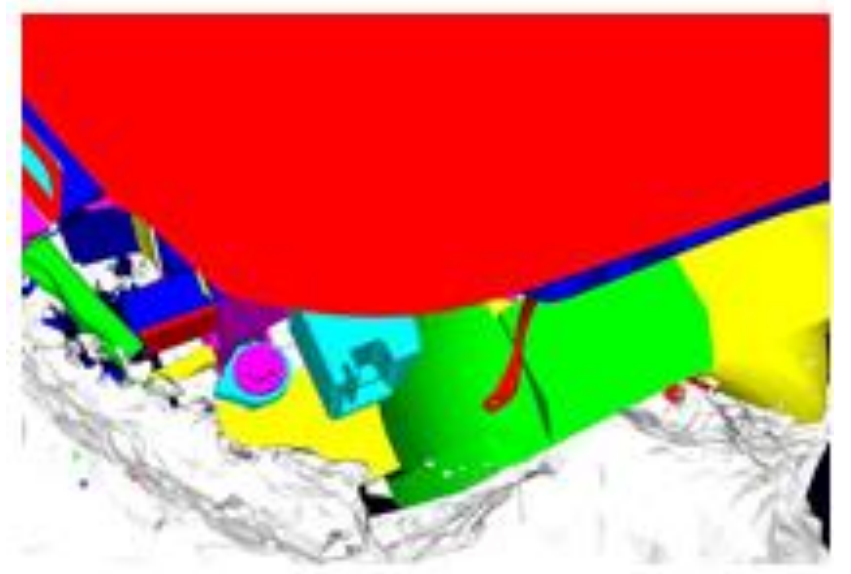

Figure 6. Side mud penetration due to the lack of duckbill protection at simulation time of 4 seconds

The main mud flow comes to the CRFM from the front fascia openings, mainly by the lower opening due to the $300 \mathrm{~mm}$ height, shown on Figure 7. 


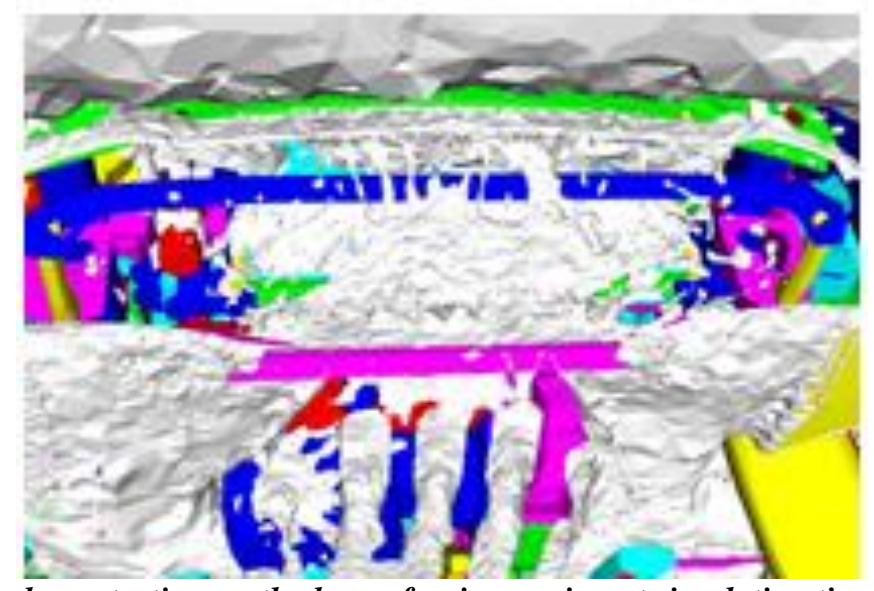

Figure 7. Mud penetration on the lower fascia opening at simulation time of 4 seconds

The methodology can be applied to evaluate CRFM performance due to mud deposition at early stages of the project, which could lead to better designs for parts and systems before any physical test is performed

\section{Complementary Studies}

As a complementary study for this work, the pressure due to mud acting on certain parts of the vehicle could evaluated in order to identify the pressure contour and magnitude over the car, similar to the one presented by BUSCARIOLO and VOLPE (2014) [2].

Another complementary work could be a correlation for the pressure loads considering both physical and test would be a great work, once this correlation may lead to evaluate structural performance of the part submitted to the mud pressure load. And for this case, it could help on improve both vehicle's development time and cost.

Another complementary work would be a use of a coupled CFD-CAE solver or Direct FSI in order to evaluate parts deformation together with the CFD multiphase simulation in order to improve more the confidence level of the simulation.

\section{REFERENCES}

1. BUSCARIOLO, F.F.; KARBON, K.J., Comparative CFD Analysis Between Rotating and Static Cases of Different Wheels Opening Designs over a Performance Sedan, SAE Paper, No 2011-36-0271, Society of Automotive Engineers, 2011.

2. BUSCARIOLO, F.F.; VOLPE, L.J.D., Water Ingestion and Pressure analysis of Automotive Vehicles using Multiphase CFD, SAE Paper, No 2014-36-0251, Society of Automotive Engineers, 2014.

3. FLUENT 14.0., User’s Guide, Fluent Inc., 2011.

4. HUCHO, W. H., Aerodynamics of Road Vehicles, From Fluid Mechanics of Vehicle Engineering, 4th Edition, SAE International, 1998. 
5. Hypermesh 11.0 User's manual Altair Computing, Inc., 2011.

6. KATZ, J., Race Car Aerodynamics: Designing for Speed, Bentley Publishers, 1995.

7. KELLY, K. B.; PROVENCHER, L. G.; SCHENKEL, F. K., The General Motors Engineering Staff Aerodynamics Laboratory - A full Scale Automotive Wind Tunnel, SAE Paper, N 820371, Society of Automotive Engineers, 1982.

8. WHITE, F., Fluid Mechanics - fouth edition, McGraw Hill, 1999

9. WIEDEMANN, J., The Influence of Ground Simulation and Wheel Rotation on Aerodynamic Drag Optimization - Potential for Reducing Fuel Consumption, SAE Paper, No 960672, Society of Automotive Engineers, 1996. 